\title{
Electromagnetic self-force on a static charge in Schwarzschild-de Sitter spacetimes
}

\author{
Joseph Kuchar ${ }^{1}$, Eric Poisson ${ }^{1}$, and Ian Vega ${ }^{1,2}$ \\ ${ }^{1}$ Department of Physics, University of Guelph, Guelph, Ontario, N1G 2W1, \\ Canada \\ 2 SISSA, Via Bonomea 265, 34136, Trieste, Italy \\ E-mail: jkuchar@uoguelph.ca \\ E-mail: epoisson@uoguelph.ca \\ E-mail: ian.vega@sissa.it
}

\begin{abstract}
We compute the self-force acting on an electric charge at rest in Schwarzschild-de Sitter spacetimes, allowing the cosmological constant to be either positive or negative. In the case of a positive cosmological constant, we show that the self-force is always positive, representing a repulsion from the black hole, and monotonically decreasing with increasing distance from the black hole. The spectrum of results is richer in the case of a negative cosmological constant. Here the self-force is not always positive - it is negative when the black-hole and cosmological scales are comparable and the charge is close to the black hole and not always monotonically decreasing - it is actually monotonically increasing when the cosmological scale is sufficiently small compared to the black-hole scale. The self-force also approaches a constant asymptotic value when the charge is moved to large cosmological distances; this feature can be explained in terms of an interaction between the charge and the conformal boundary at infinity, which acts as a grounded conductor.
\end{abstract}

PACS numbers: 04.20.-q, 04.40.-b, 41.20.Cv

\section{Introduction}

To hold a particle in place in the static spacetime of a massive body requires an external force, which compensates for the body's gravity. For a given spacetime, the required force depends on the nature of the particle; in particular, the force differs when the particle carries an electric charge, relative to its value when the particle is neutral. The difference accounts for the particle's self-force, an effect that originates in the way that the particle's electromagnetic field interacts with the spacetime curvature. This interaction is subtle, it involves remote portions of the spacetime, and as a result the self-force is notoriously unintuitive; it is virtually impossible to predict even the sign of the self-force before it is revealed by a detailed calculation. For example, it is known from the pioneering work of Smith and Will [1] that the self-force on a particle at rest in the Schwarzschild spacetime of a nonrotating black hole is repulsive, which implies a smaller external force relative to a neutral particle; this outcome has defied attempts to provide an intuition. (For a valiant attempt, refer to Sec. IV of Ref. [2].)

The study of self-forces in curved spacetime was initiated by DeWitt and Brehme [3, and there is currently a large effort (reviewed in Ref. 4]) devoted to 
the computation of self-forces in various spacetimes. Most of the recent activity has focused on the gravitational self-force, in an effort to model the inspiral and gravitational-wave emissions of extreme mass-ratio binaries [5, 6, 7]. Our concern here is elsewhere. We have unfinished business with even the simplest exemplars of self-forces in curved spacetimes, those involving static particles outside massive bodies. We are troubled by the lack of intuition surrounding these simple situations, and have pursued a line of inquiry that aims to probe more deeply into the mysterious aspects of the self-force. We intend to achieve this by producing a larger catalogue of self-forces, examining its entries carefully, and extracting whatever intuition can be extracted.

Previous studies in this program have focused on altering the nature of the massive body, replacing the black hole of the Smith-Will work with a material body of some kind. Thus, Burko, Liu and Soen [2, building on earlier work by Unruh [8, replaced the black hole by a massive thin shell. Shankar and Whiting 9 considered a body of constant density, and Isoyama and Poisson [10] calculated the self-force outside relativistic polytropes. In all cases the results confirmed the analysis of Drivas and Gralla 11, who considered static charges in the exterior spacetime of a spherical body of arbitrary composition. In particular, Drivas and Gralla expanded the selfforce in powers of $r_{0}^{-1}$, where $r_{0}$ is the radial position of the charge, and showed that the leading term at order $r_{0}^{-3}$ is universal, independent of the body's internal composition. The universal self-force is given by the Smith-Will expression $e^{2} M / r_{0}^{3}$, where $e$ is the particle's charge and $M$ the mass of the body. They also showed that the dependence of the self-force on details of internal composition is revealed by the subdominant terms at order $r_{0}^{-5}$ and beyond; these were examined closely by Isoyama and Poisson, who showed how the self-force can be used as a probe of the body's internal structure.

In this paper we place our focus elsewhere. Instead of altering the nature of the massive body, we alter the nature of the asymptotic spacetime. We calculate the self-force acting on a charged particle held in place outside a black hole in a de Sitter universe. We allow the cosmological constant to be either positive (de Sitter asymptotics) or negative (anti de Sitter asymptotics). So while the massive body is now fixed to be a black hole, the spacetime is no longer asymptotically flat, and our goal is to determine the impact of this change on the electromagnetic self-force.

The immersion of the black hole in a de Sitter universe produces a spacetime with two fundamental length scales. The first is the event-horizon radius $r_{e}$, and the second is the cosmological scale $r_{c}$; in the case of the Schwarzschild-de Sitter spacetime, $r_{c}$ is also the radius of the cosmological horizon. The ability to tune $r_{c}$ in relation to $r_{e}$ offers interesting possibilities for self-force calculations, and broadens the range of situations for which one might seek an intuition. To begin we may set $r_{e} \ll r_{c}$ and consider spacetimes for which there is a clean separation of scales. In such a situation we might expect that the self-force is mostly sensitive to the Schwarzschild aspects of the spacetime when $r_{0} \ll r_{c}$, and mostly sensitive to its de Sitter aspects when $r_{0} \gg r_{e}$.

In the case of Schwarzschild-de Sitter spacetime, this means that the self-force should reduce to the Smith-Will expression when $r_{0} \ll r_{c}$, while it should approach zero when $r_{0} \gg r_{e}$, possibly faster than $r_{0}^{-3}$. This is because de Sitter spacetime is conformally flat and the electromagnetic field is conformally invariant, leading to a vanishing self-force in pure de Sitter spacetime. The situation is more subtle in Schwarzschild-anti de Sitter spacetime, because of the presence of a conformal boundary at $r=\infty$. When $r_{0} \ll r_{c}$ the self-force should still be well approximated 
by the Smith-Will expression, but when $r_{0} \gg r_{e}$ it should be conformally related to the force experienced by a particle in a truncated flat spacetime.

It is good news for intuition-building that these expectations are borne out by our computations. The interesting cases, however, are produced when the scales $r_{e}$ and $r_{c}$ are not widely separated. In such a situation there is no Schwarzschild regime when $r_{0} \ll r_{c}$, and there is no de Sitter regime when $r_{0} \gg r_{e}$. Instead the self-force can be expected to depend on all aspects of the spacetime, and one would be hard-pressed to predict the outcome of a detailed computation. And indeed, we shall find some surprising features when $r_{e}, r_{0}$, and $r_{c}$ are all comparable to each other.

We explain how the electromagnetic self-force is computed in Sec. 2, and describe the results of our numerical calculations in Sec. 3 . In Sec. 4 we investigate the behaviour of the self-force in Schwarzschild-anti de Sitter spacetime by formulating a simple approximation that can be treated analytically, and showing that it reproduces our numerical results when $r_{0} \gg r_{c}$. We offer some concluding remarks in Sec. 5. and in the Appendix we explain why we refrain from computing the self-force on a scalar charge in Schwarzschild-de Sitter spacetimes.

\section{Self-force computations}

\subsection{Spacetime}

We work with the class of Schwarzschild-de Sitter spacetimes, with a metric described by

$$
d s^{2}=-f d t^{2}+f^{-1} d r^{2}+r^{2} d \Omega^{2},
$$

with

$$
f=1-\frac{2 M}{r}-\frac{1}{3} \Lambda r^{2}
$$

and $d \Omega^{2}=d \theta^{2}+\sin ^{2} \theta d \phi^{2}$. The spacetime contains a black hole of mass $M$, and the metric is a solution to the Einstein field equations with cosmological constant $\Lambda$. We allow $\Lambda$ to be either positive or negative; when $\Lambda<0$ the solution is often referred to as the Schwarzschild-anti de Sitter metric.

When $\Lambda>0$ the spacetime contains both an event horizon at $r=r_{e}$ and a cosmological horizon at $r=r_{c}$. It is useful to adopt $r_{e}$ and $r_{c}$ as the primary parameters of the spacetime, and to express $M$ and $\Lambda$ as functions of these parameters. This is achieved by factorizing $f$ as $f=-k\left(r-r_{e}\right)\left(r-r_{c}\right)\left(r+r_{e}+r_{c}\right) / r$ and comparing with Eq. (2.2). We get

$$
2 M=\frac{r_{e} r_{c}\left(r_{e}+r_{c}\right)}{r_{c}^{2}+r_{e} r_{c}+r_{e}^{2}}, \quad \Lambda=\frac{3}{r_{c}^{2}+r_{e} r_{c}+r_{e}^{2}} .
$$

When $r_{e} \ll r_{c}$ these relations reduce to $2 M \simeq r_{e}$ and $\Lambda \simeq 3 / r_{c}^{2}$.

When $\Lambda<0$ the spacetime still contains an event horizon at $r=r_{e}$, but there is no longer a cosmological horizon. The spacetime possesses anti de Sitter asymptotics, with a timelike conformal boundary at $r=\infty$. In this case also it is useful to use $r_{e}$ and a cosmological length scale $r_{c}$ to parametrize the spacetime. The definition of $r_{c}$ is somewhat arbitrary, and we fix it with the factorization $f=-k\left(r-r_{e}\right)\left(r^{2}+r_{e} r+r_{c}^{2}\right) / r$. This produces the alternative relations

$$
2 M=\frac{r_{e} r_{c}^{2}}{r_{c}^{2}-r_{e}^{2}}, \quad \Lambda=-\frac{3}{r_{c}^{2}-r_{e}^{2}}
$$


for the mass and cosmological constant. When $r_{e} \ll r_{c}$ we still have $2 M \simeq r_{e}$ and $\Lambda \simeq-3 / r_{c}^{2}$.

\subsection{Electromagnetic self-force}

The equations that permit a computation of the self-force on a charge at rest in a static, spherically-symmetric spacetimes were developed in Sec. X of Ref. 12. We provide a brief summary, specializing their results to the specific case of a Schwarzschild-de Sitter spacetime.

The electromagnetic field tensor $F_{\alpha \beta}$ is expressed as $F_{\alpha \beta}=\nabla_{\alpha} A_{\beta}-\nabla_{\beta} A_{\alpha}$ in terms of a potential $A_{\alpha}$, and in the Lorenz gauge $\nabla_{\alpha} A^{\alpha}=0$ the complete set of Maxwell equations reduce to

$$
\square A^{\alpha}-R_{\beta}^{\alpha} A^{\beta}=-4 \pi j^{\alpha},
$$

in which $\square=g^{\alpha \beta} \nabla_{\alpha} \nabla_{\beta}$ is the wave operator in curved spacetime, $R_{\beta}^{\alpha}$ is the Ricci tensor, and $j^{\alpha}$ is the current density. For a point particle moving on a world line described by the parametric equations $x^{\alpha}=z^{\alpha}(\tau)$, where $\tau$ is proper time, the current density at the spacetime event $x$ is

$$
j^{\alpha}(x)=e \int u^{\alpha} \delta(x, z(\tau)) d \tau
$$

in which $e$ is the particle's electric charge, $u^{\alpha}=d z^{\alpha} / d \tau$ is the velocity vector, and $\delta\left(x, x^{\prime}\right)$ is a scalarized delta function. In our developments here, the particle is placed on a static world line described by $r=r_{0}$.

A computation of the self-force involves the component $A_{t}$ only, and a development in spherical harmonics,

$$
A_{t}(r, \theta, \phi)=\sum_{\ell m} R_{\ell m}(r) Y_{\ell m}(\theta, \phi),
$$

turns Eq. 2.5 into an ordinary second-order differential equation for the radial functions $R_{\ell m}$. By placing the particle on the polar axis $\theta=0$ we ensure that modes with $m \neq 0$ vanish, and we obtain

$$
r^{2} R_{\ell}^{\prime \prime}+2 r R_{\ell}^{\prime}-\frac{\ell(\ell+1)}{f} R_{\ell}=4 \pi e \sqrt{\frac{2 \ell+1}{4 \pi}} \delta\left(r-r_{0}\right)
$$

for the nonvanishing modes $R_{\ell}:=R_{\ell 0}$; a prime indicates differentiation with respect to $r$.

Near a horizon at $f=0$, Eq. (2.8) admits the asymptotic expansion $R=$ $R_{h} f\left(1+R_{1} f+R_{2} f^{2}+\cdots\right)$, in which the coefficients $R_{1}, R_{2}$, and so on can be determined from the differential equation; the overall normalization $R_{h}$ is arbitrary. This expression can be used to set the values of $R_{\ell}$ and $R_{\ell}^{\prime}$ for a numerical integration of the equation that starts at $r=r_{e}(1+\epsilon)$ with $\epsilon \ll 1$. The strategy works also for an integration starting at $r=r_{c}(1-\epsilon)$, when the spacetime contains a cosmological horizon. For anti de Sitter asymptotics the differential equation produces the asymptotic behaviours $e^{ \pm s / r}$, where $s^{2}:=3 \ell(\ell+1) /|\Lambda|$. Both solutions are bounded in the limit $r \rightarrow \infty$, and to make the problem well-posed we impose the Dirichlet boundary condition $A_{t}(\infty, \theta, \phi)=0$ on the potential. (This can be thought of as the static limit of reflecting-wave boundary conditions at $r=\infty$.) The selected asymptotic form is therefore $R \sim R_{\infty}\left(e^{s / r}-e^{-s / r}\right)$, and this can be used to set the 
values of $R_{\ell}$ and $R_{\ell}^{\prime}$ for a numerical integration that starts at $r=r_{\infty} \gg r_{c}$; the overall normalization $R_{\infty}$ is arbitrary.

The integration of Eq. 2.8) proceeds outward from $r=r_{e}(1+\epsilon)$ with an undetermined normalization $R_{e}$, and it simultaneously proceeds inward from $r=$ $r_{c}(1-\epsilon)$ or $r=r_{\infty}$ with another undetermined normalization $R_{c}$ or $R_{\infty}$. The normalizations are determined by enforcing the junction conditions

$$
\left[R_{\ell}\right]=0, \quad\left[R_{\ell}^{\prime}\right]=\frac{4 \pi e}{r_{0}^{2}} \sqrt{\frac{2 \ell+1}{4 \pi}}
$$

at $r=r_{0}$, where $[\psi]=\psi\left(r_{0}^{+}\right)-\psi\left(r_{0}^{-}\right)$denotes the jump of a quantity $\psi$ across $r=r_{0}$; in an obvious notation, $r_{0}^{ \pm}=r_{0}(1 \pm \epsilon)$ with $\epsilon>0$, and the jump is evaluated in the limit $\epsilon \rightarrow 0$.

The equation for $\ell=0$ admits an analytical solution. The general solution to the homogeneous equation is $R_{0}=a+b / r$, where $a$ and $b$ is a constant. The constant solution has no impact on the self-force, and enforcing the junctions conditions, we have that $R_{0}=-\sqrt{4 \pi} e / r$ for $r>r_{0}$, and $R_{0}=-\sqrt{4 \pi} e / r_{0}$ for $r<r_{0}$.

The radial functions can next be involved in a mode-sum computation of the electromagnetic self-force. The required expression can be obtained from Eqs. (10.10), (10.22), and (10.23) of Ref. [12]. We have

$F^{r}=e^{2} f_{0} \sum_{\ell=0}^{\infty}\left[\sqrt{\frac{2 \ell+1}{4 \pi}} \frac{R_{\ell}^{\prime}\left(r_{0}+\Delta\right)}{e f_{0}^{1 / 2}}-A\left(\ell+\frac{1}{2}\right)-B-\frac{D}{\left(\ell-\frac{1}{2}\right)\left(\ell+\frac{3}{2}\right)}-\cdots\right]$,

in which $f_{0}=f\left(r_{0}\right)$ and $A, B$, and $D$ are regularization parameters that permit the mode-by-mode subtraction of the singular part of the electromagnetic field (the method originates in the work of Barack and Ori; see Refs. [13, 14, 15]). Explicit expressions are given in Eq. (10.24) of Ref. [12, and for the specific case of Schwarzschild-de Sitter spacetime the regularization parameters are given by

$$
\begin{aligned}
A & =\frac{1}{r_{0}^{2} f_{0}^{1 / 2}} \operatorname{sign}(\Delta), \\
B & =-\frac{1}{2 r_{0}^{3} f_{0}}\left(3 M-r_{0}\right), \\
D & =\frac{1}{48 r_{0}^{5} f_{0}^{2}}\left(45 M^{3}-27 M^{2} r_{0}+24 M^{2} \Lambda r_{0}^{3}-9 M \Lambda r_{0}^{4}+3 \Lambda r_{0}^{5}-4 M \Lambda^{2} r_{0}^{6}\right) .
\end{aligned}
$$

In Eq. (2.10) the derivative of the radial function is evaluated at $r=r_{0}+\Delta$ in the limit $\Delta \rightarrow 0$; the limit can be taken from either direction, and the sign of $A$ reflects this choice.

\subsection{Numerical implementation}

The differential equation (2.8) was integrated numerically using the routine odeint of the SciPy (Scientific Python) library. For Schwarzschild-de Sitter spacetime the equation is integrated forward from $r=r_{e}(1+\epsilon)$ and backward from $r=r_{c}(1-\epsilon)$, using starting values obtained from an asymptotic analysis. For Schwarzschild-anti de Sitter spacetime the backward integration proceeds instead from $r=r_{\infty} \gg r_{c}$, with appropriate starting values. In both cases the solutions are matched at $r=r_{0}$ by enforcing the junction conditions.

The radial functions $R_{\ell}^{\prime}\left(r_{0}\right)$ are then inserted within Eq. 2.10 to evaluate the electromagnetic self-force. The regularization procedure, which involves subtracting 
the $A, B$, and $D$ terms from the radial function, is a powerful diagnostic of numerical accuracy. Examining Eq. 2.10), we observe that a plot of the bracketed quantity as a function of $\ell$ must reveal a $\ell^{-4}$ falloff when $\ell$ is large; failure to obtain this behaviour signals either coding errors or issues of numerical stability. All results presented below have been validated by a careful monitoring of such multipole plots.

The mode sum of Eqs. 2.10 is typically truncated at $\ell=\ell_{\max }=80$. Additional accuracy can be obtained by estimating the remaining portion of the sum [16. If we denote each term in the mode sum by $F_{\ell}^{r}$, then the self-force is calculated as $F^{r}=$ data + tail, with data given by the partial mode sum up to $\ell=\ell_{\max }$, and tail by the remaining sum from $\ell_{\max }+1$ to infinity. There is no numerical data to calculate the tail, but when $\ell_{\max }$ is sufficiently large, $F_{\ell}^{r}$ can be approximated by [16]

$$
\frac{E}{\left(\ell-\frac{3}{2}\right)\left(\ell-\frac{1}{2}\right)\left(\ell+\frac{3}{2}\right)\left(\ell+\frac{5}{2}\right)} \text {. }
$$

Summing over this estimate yields

$$
\text { tail } \simeq \frac{16 E}{3} \frac{\ell_{\max }+1}{\left(2 \ell_{\max }-1\right)\left(2 \ell_{\max }+1\right)\left(2 \ell_{\max }+3\right)\left(\ell_{\max }+5\right)} .
$$

The coefficient $E$ is unknown, but it can be estimated from the last data point at $\ell=\ell_{\max }$

$$
E \simeq\left(\ell_{\max }-\frac{3}{2}\right)\left(\ell_{\max }-\frac{1}{2}\right)\left(\ell_{\max }+\frac{3}{2}\right)\left(\ell_{\max }+\frac{5}{2}\right) F_{\ell_{\max }}^{r} .
$$

Making the substitution returns the estimate

$$
\text { tail } \simeq \frac{\left(2 \ell_{\max }-3\right)\left(\ell_{\max }+1\right)}{3\left(2 \ell_{\max }+1\right)} F_{\ell_{\max }}^{r}
$$

for the tail. Adding this to data improves the final result for the self-force, and provides a gross overestimate of the numerical accuracy. With $\ell_{\max }=80$ we find that the error bars on $F^{r}$ would not be visible on the plots displayed in Sec. 3. A more accurate estimation of tail [16] uses additional points near $\ell=\ell_{\max }$ to fit for additional parameters $F, G, H$, which are then included with appropriate polynomials in tail; we do not pursue such refinements here.

\section{Self-force results}

\subsection{Schwarzschild-de Sitter spacetime}

We have performed a range of self-force computations for a static particle in Schwarzschild-de Sitter spacetime. The computations have three input parameters: the event-horizon radius $r_{e}$, the particle's position $r_{0}$, and the cosmological radius $r_{c}$; these are restricted by $r_{e}<r_{0}<r_{c}$. It is convenient to rescale all lengths by $r_{e}$, which can be thought of as the fundamental scale of the spacetime. Because $r_{e} \simeq 2 M$ when $r_{e} \ll r_{c}$, we find it helpful to set $r_{e}=2$ in our computations, loosely thinking of the mass of the black hole as setting the unit of length. The particle's charge $e$ is not an essential input parameter, because the self-force necessarily scales as $e^{2}$; for our computations we set $e=1$.

A sample of our results are presented in Fig. 1 for each selected value of $r_{c}$ we display $F:=f_{0}^{-1 / 2} F^{r}$ as a function of $r_{0}$. The division by $\sqrt{f_{0}}$, where $f_{0}:=f\left(r_{0}\right)$, translates $F^{r}$ into a coordinate-invariant quantity. For example, $F$ is the self-force as measured by a static observer at $r=r_{0}$, making use of an orthonormal frame there. 

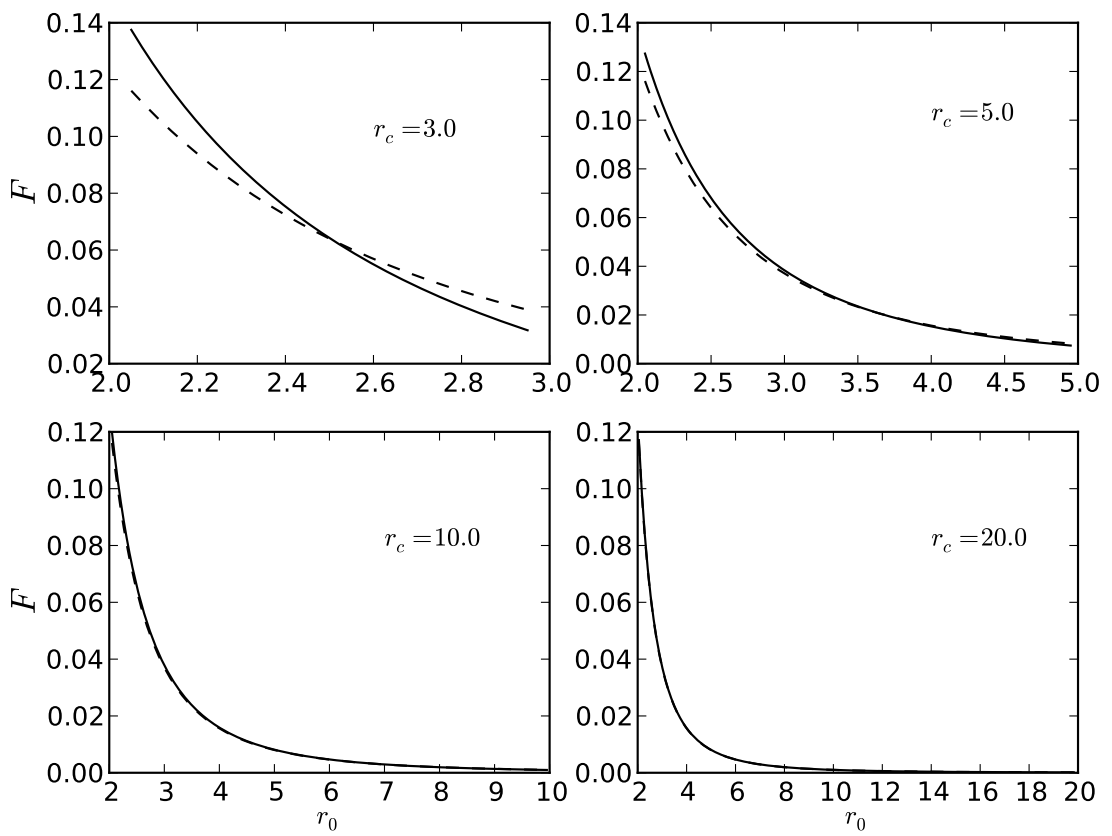

Figure 1. Electromagnetic self-force $F=F^{r} / \sqrt{f_{0}}$ acting on a static charge in Schwarzschild-de Sitter spacetime. In all cases $r_{e}=2$ in arbitrary units, and computations are shown as a solid curve for selected values of $r_{c}$. The dashed curve represents the Smith-Will force for a black hole of mass $M=1$. The solid and dashed curves are barely distinguishable when $r_{c}=10$ and $r_{c}=20$.

Alternatively, $F$ is the square root of the spacetime invariant $g_{\alpha \beta} F^{\alpha} F^{\beta}$, with a choice of sign inherited from $F^{r}$. An immediate observation from Fig. 1 is that $F>0$ in all cases; the electromagnetic self-force always represents a repulsion from the black hole. A second observation is that the self-force is monotonically decreasing as a function of $r_{0}$.

To reflect on these results it is helpful to begin with cases for which $r_{c} \gg r_{e}$. In such situations we can expect that an electric charge at $r_{0} \ll r_{c}$ will feel a selfforce that is mostly sensitive to the black-hole aspects of the spacetime, and largely insensitive to the de Sitter asymptotic conditions. We would therefore expect the self-force to be well approximated by the Smith-Will expression [1]

$$
F=\frac{e^{2} M}{r_{0}^{3}}
$$

in which $M$ is well approximated by $r_{e} / 2$. On the other hand, an electric charge at $r_{0} \gg r_{e}$ should feel a self-force that is mostly sensitive to the de Sitter aspects of the spacetime. Because de Sitter spacetime is conformally flat, and because Maxwell's equations are conformally invariant, the self-force should vanish in this limit. Combining these observations, we would expect that the self-force should display a change of behaviour as $r_{0}$ increases from $r_{e}$ toward $r_{c}$; the Smith-Will behaviour should gradually give way to a self-force that approaches zero faster than $r_{0}^{-3}$. 

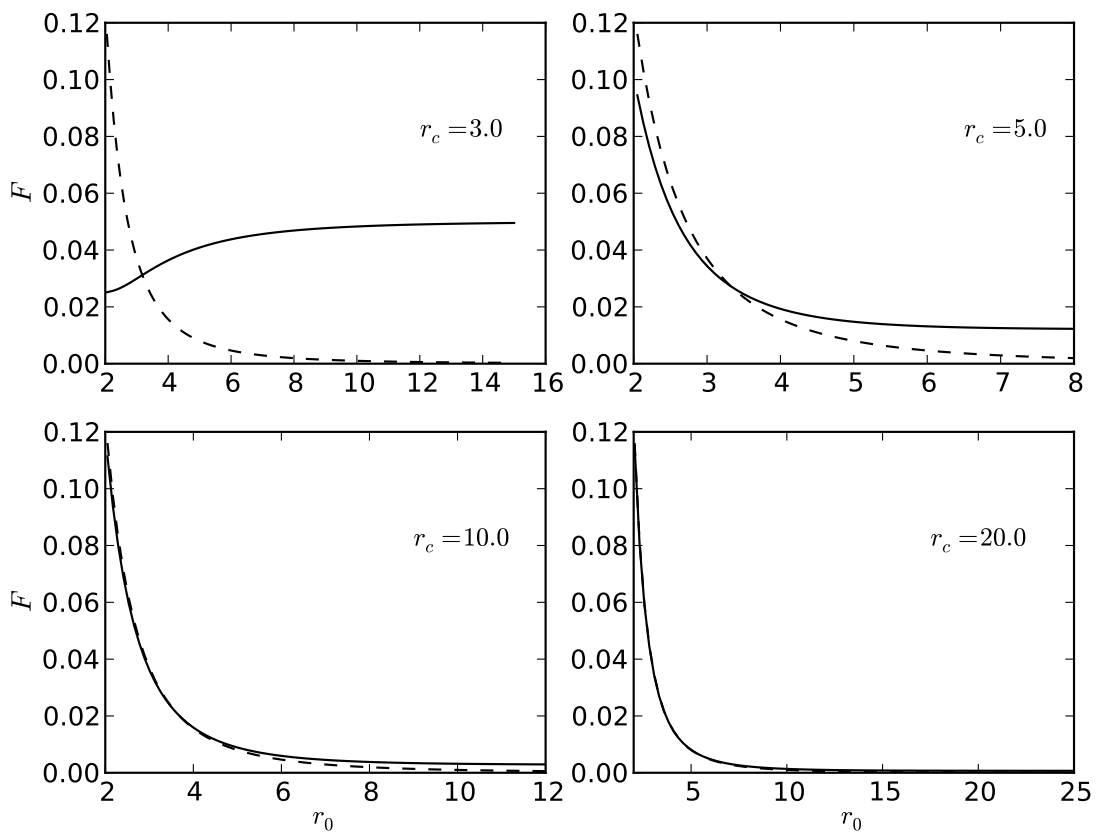

Figure 2. Electromagnetic self-force $F=F^{r} / \sqrt{f_{0}}$ acting on a static charge in Schwarzschild-anti de Sitter spacetime. In all cases $r_{e}=2$ in arbitrary units, and computations are shown as a solid curve for selected values of $r_{c} \geq 3$. The dashed curve represents the Smith-Will force for a black hole of mass $M=1$. The solid and dashed curves are barely distinguishable when $r_{c}=20$.

These expectations are borne out by the numerical results. We see generally speaking that when $r_{e} \ll r_{c}$, the self-force is well approximated by the Smith-Will expression when $r_{0}$ is small, and that the force becomes smaller than this when $r_{0}$ becomes comparable to $r_{c}$. The difference between $F$ and the Smith-Will force is very slight when $r_{c} \gg r_{e}$, but it increases as $r_{c}$ is decreased toward $r_{e}$; in this regime there is no longer a clean separation of scales, and the self-force is sensitive to all aspects of the spacetime. As $r_{c}$ becomes comparable to $r_{e}$ we see that the Smith-Will expression underestimates the self-force when $r_{0}$ is close to $r_{e}$, and overestimates it when $r_{0}$ is close to $r_{c}$. The discrepancy, however, is never worse than approximately $15 \%$, which allows to conclude that the Smith-Will expression always provides an adequate approximation to the self-force.

\subsection{Schwarzschild-anti de Sitter spacetime}

We have also performed a range of self-force computations for a static charge in Schwarzschild-anti de Sitter spacetime. Here also the computations have three input parameters: the event-horizon radius $r_{e}$, the particle's position $r_{0}$, and the cosmological length scale $r_{c}$; in this case it is possible for $r_{0}$ to exceed $r_{c}$. Once more we set $r_{e}=2$ in our computations.

A sample of our results are presented in Figs. 2 and 3 . The figures reveal the 

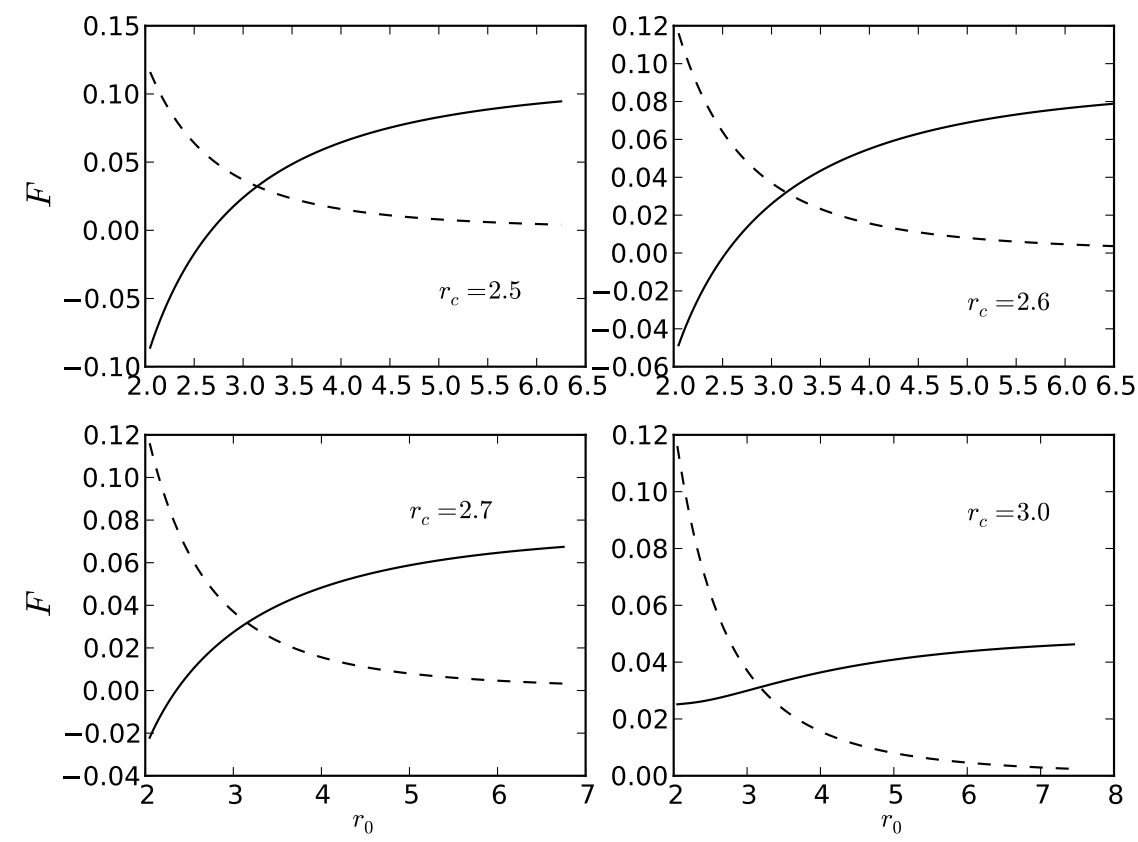

Figure 3. Electromagnetic self-force acting on a static charge in Schwarzschildanti de Sitter spacetime. In all cases $r_{e}=2$ in arbitrary units, and computations are shown for $r_{c} \leq 3$.

following features. First, $F>0$ whenever $r_{c}>3$, so that in this regime the selfforce represents a repulsion from the black hole. Second, we notice that $F<0$ when $r_{0}<r_{c}<2.9$, so that in this regime the self-force actually represents an attraction toward the black hole. Third, the self-force is well approximated by the Smith-Will expression when $r_{c} \gg r_{e}$, but only when $r_{0}$ is smaller than $r_{c}$; when $r_{0}>r_{c}$ we see the self-force approaching a constant asymptotic value $F_{\infty}$, while the Smith-Will expression continues to decay as $r_{0}^{-3}$. Fourth, when $r_{c}$ is comparable to $r_{e}$, the Smith-Will expression significantly overestimates the self-force when $r_{0}<r_{c}$, and it significantly underestimates it when $r_{0}>r_{c}$. And fifth, when $r_{c}>4.9$ the selfforce is monotonically decreasing toward $F_{\infty}$ as $r_{0}$ increases toward infinity, while it is monotonically increasing toward $F_{\infty}$ when $r_{c}<3.0$; when $3.0<r_{c}<4.9$ the self-force is no longer monotonic, reaching a minimum value at some $r_{0}$ before increasing again (this behaviour is not shown in the figures).

The existence of a regime $r_{0}<r_{c}<2.9$ giving rise to an attractive self-force is a surprising feature of Schwarzschild-anti de Sitter spacetime; there is no intuitive explanation for this outcome. Another surprising feature is the approach to a constant asymptotic value $F_{\infty}$ when $r_{0} \gg r_{c}$. Our numerical results indicate that when $r_{e} \ll r_{c}$, $F_{\infty}$ is extremely well approximated by the expression

$$
F_{\infty}=\frac{e^{2}}{4 r_{c}^{2}}
$$

so that it decreases with increasing $r_{c}$ - this behaviour can be gleaned from Fig. 2. As 
we shall see in Sec.4. it is actually easy, after the fact, to explain this result. Another regime that comforts intuition is the one with $r_{e} \ll r_{c}$ and $r_{0} \ll r_{c}$, in which the selfforce is dominated by the black-hole aspects of the spacetime, and well approximated by the Smith-Will expression.

\section{Electromagnetic self-force in anti de Sitter spacetime}

In this section we shed some light on the asymptotic limit of Eq. (3.2) by considering a static charge at a radius $r_{0} \gg r_{c}$ in Schwarzschild-anti de Sitter spacetime. In this portion of the spacetime the metric is well approximated by

$$
d s^{2} \simeq \frac{r^{2}}{a^{2}}\left(-d t^{2}+\frac{a^{4}}{r^{2}} d r^{2}+a^{2} d \Omega^{2}\right),
$$

where $a^{2}:=3 /|\Lambda| ;$ when $r_{e} \ll r_{c}$ we have that $a \simeq r_{c}$. The coordinate transformation $\rho=a(1-a / r)$ brings the metric to the form

$$
d s^{2} \simeq \frac{r^{2}}{a^{2}}\left(-d t^{2}+d \rho^{2}+a^{2} d \Omega^{2}\right)
$$

in which $r$ is to be expressed as a function of $\rho$. The spacetime is approximately conformal to a spacetime with metric

$$
d \tilde{s}^{2}=-d t^{2}+d \rho^{2}+a^{2} d \Omega^{2},
$$

and the self-force on the static charge can be calculated by working in the conformal spacetime.

The conformal boundary is situated at $\rho=a$, and the conformal spacetime is necessarily truncated at this limiting value. The Dirichlet condition $A_{t}(r=\infty, \theta, \phi)=$ 0 imposed on the potential implies that the boundary acts as a grounded conductor. When $r_{0} \gg a$ we have that $\rho_{0}=a\left(1-a / r_{0}\right)$ is very close to $a$, so that the charge is in the immediate vicinity of the conductor. The electric field produced by the charge is therefore strongly influenced by the conducting surface, and we expect that it can be computed by exploiting the method of images. We also expect the self-force to arise as a consequence of the interaction between the charge and its image.

To carry out the calculations we work in a small coordinate patch around the charge. The patch is centered at $\left(\rho_{0}, \theta_{0}, \phi_{0}\right)$, and because the $\rho$-dependence of the electric field is the central aspect of the problem, we allow ourselves to approximate the angular part of the line element as

$$
a^{2} d \Omega^{2} \simeq a^{2} d\left(\theta-\theta_{0}\right)^{2}+a^{2} \sin ^{2} \theta_{0} d\left(\phi-\phi_{0}\right)^{2}=d x^{2}+d y^{2},
$$

where $x=a\left(\theta-\theta_{0}\right)$ and $y=a \sin \theta_{0}\left(\phi-\phi_{0}\right)$ are locally Cartesian coordinates. With this simplification the conformal metric becomes

$$
d \tilde{s}^{2}=-d t^{2}+\rho^{2}+d x^{2}+d y^{2},
$$

and the conformal boundary at $\rho=a$ becomes flat.

This situation is now elementary. We have a charge $e$ at $\rho=\rho_{0}$, a flat conductor at $\rho=a$, and an image charge $-e$ at a position $\rho=a+\left(a-\rho_{0}\right)$ beyond the conductor. The distance between the charges is $d=2\left(a-\rho_{0}\right)=2 a^{2} / r_{0}$, and the electric field supplied by the image charge is equal to $E=e / d^{2}=e r_{0}^{2} /\left(4 a^{4}\right)$ when evaluated at the physical charge; the field points in the positive $\rho$ direction, toward the image charge. The electromagnetic field tensor is $\tilde{F}_{t \rho}=-E$ in the conformal spacetime. 
Because Maxwell's equations are conformally invariant, we have that $F_{\alpha \beta}=\tilde{F}_{\alpha \beta}$, and our previous results imply that $E=-F_{t \rho}$ is also the electric field in the physical spacetime. Transforming back to the original radial coordinate, we have that

$$
F_{t r}=\frac{d \rho}{d r} F_{t \rho}=-\frac{e}{4 a^{2}} .
$$

The force $F^{\alpha}=e F_{\beta}^{\alpha} u^{\beta}$ exerted by this electric field evaluates to $F^{r}=e^{2} f_{0}^{1 / 2} /\left(4 a^{2}\right)$, or

$$
F=\frac{e^{2}}{4 a^{2}}
$$

after division by $\sqrt{f_{0}}$ to convert $F^{r}$ into the invariant $F$. When $r_{e} \ll r_{c}$ this reduces to $F=e^{2} /\left(4 r_{c}^{2}\right)$, and this is precisely what was observed in Sec. 3.2 . We see that the constant asymptotic behaviour is explained very convincingly in terms of an interaction between the charge and the conducting surface that must be placed at the conformal boundary to enforce the Dirichlet boundary conditions. While the relation of Eq. (3.2) was first obtained by means of a fit to our numerical results, we see that the value of $F_{\infty}$ is revealed by a very simple analytical calculation.

\section{Concluding remarks}

We have computed the self-force acting on an electric charge at rest in Schwarzschild-de Sitter spacetimes, allowing the cosmological constant to be either positive or negative. Our results reveal some intuitive features, but they also display aspects that defy intuition.

In the case of a positive cosmological constant, we have seen that the self-force is always positive, representing a repulsion from the black hole, and monotonically decreasing with increasing $r_{0}$. When $r_{e} \ll r_{c}$, so that the black-hole and cosmological scales are well separated, the self-force is dominated by the black-hole aspects of the spacetime when $r_{0} \ll r_{c}$, and it is well approximated by the Smith-Will expression of Eq. 3.1); it falls off faster than the Smith-Will expression when $r_{0} \gg r_{e}$, where the de Sitter aspects of the spacetime are dominant. These results are intuitive, to the extent that they can be expected on the basis of the Smith-Will force (which is itself unintuitive) and the fact that the self-force necessarily vanishes in pure de Sitter spacetime. When $r_{e}$ is comparable to $r_{c}$, the mixing of scales makes this intuition unreliable. The self-force, however, continues to behave in a qualitatively similar manner, and we note that the Smith-Will expression continues to provide an adequate approximation; the discrepancy never exceeds $15 \%$ in the full range $r_{e}<r_{0}<r_{c}$.

The spectrum of results is richer in the case of a negative cosmological constant. Here the self-force is not always positive - it goes negative when $r_{0}<r_{c}<3$, in units in which $r_{e}=2$ - and it is monotonically decreasing for $r_{c}>4.9$ only - it is monotonically increasing for $r_{c}<3.0$. These features are not intuitive. One aspect that could be anticipated, however, is the asymptotic approach to a constant value $F_{\infty}$ when $r_{0} \gg r_{c}$; as we saw in Sec. 4. this is the result of an interaction between the charge and the conformal boundary at $r=\infty$, which acts as a grounded conductor.

It is comforting that some aspects of the self-force admit intuitive explanations, but it is perplexing that so many do not. This situation continues to motivate further study. 
Electromagnetic self-force on a static charge in Schwarzschild-de Sitter spacetimes 12

\section{Appendix A. Scalar self-force}

The self-force on a scalar charge $q$ in curved spacetime is often considered as a toy model for the electromagnetic self-force, and in most situations the computations of the scalar self-force are considerably simpler. For a charge at rest in a static spacetime the degree of complexity is exactly the same, because as we have seen, the computation of the electromagnetic self-force involves a single component of the vector potential $A_{\alpha}$. So with the infrastructure put in place to calculate the electromagnetic self-force, it is usually a simple matter to make the changes required to compute a scalar self-force.

Our intention was indeed to perform scalar self-force computations, until we came to realize that a static scalar charge in Schwarzschild-de Sitter spacetime produces a scalar field that is necessarily singular at one of the horizons. The conclusion extends to pure de Sitter spacetime, where the field either diverges at $r=0$ or at $r=r_{c}$. The conclusion, however, does not extend to Schwarzschild-anti de Sitter spacetime, to pure anti de Sitter spacetime, or to a scalar field that is nonminimally coupled to the spacetime curvature. Because of these issues, we have abandoned our goal to compute scalar self-forces in Schwarzschild-de Sitter spacetimes.

The problem is easily identified with an examination of the $\ell=0$ mode of the scalar equation. The scalar field $\Phi$ is taken to satisfy the wave equation

$$
\square \Phi=-4 \pi \mu,
$$

in which $\mu$ is the scalar charge density. For a point particle this is given by

$$
\mu(x)=q \int \delta(x, z(\tau)) d \tau,
$$

where $q$ is the particle's scalar charge. The particle is placed on a static world line $z(\tau)$ described by $r=r_{0}$. A decomposition in spherical harmonics,

$$
\Phi(r, \theta, \phi)=\sum_{\ell m} R_{\ell m}(r) Y_{\ell m}(\theta, \phi),
$$

gives rise to the ordinary second-order differential equation

$r^{2} R_{\ell}^{\prime \prime}+\left(2+\frac{r f^{\prime}}{f}\right) r R_{\ell}^{\prime}-\frac{\ell(\ell+1)}{f} R_{\ell}=-\frac{4 \pi q}{f_{0}^{1 / 2}} \sqrt{\frac{2 \ell+1}{4 \pi}} \delta\left(r-r_{0}\right)$

for the nonvanishing modes $R_{\ell}:=R_{\ell 0}$; a prime indicates differentiation with respect to $r$. The radial functions are required to satisfy the junction conditions

$$
\left[R_{\ell}\right]=0, \quad\left[R_{\ell}^{\prime}\right]=-\frac{4 \pi e}{r_{0}^{2} f_{0}^{1 / 2}} \sqrt{\frac{2 \ell+1}{4 \pi}}
$$

at $r=r_{0}$.

The equation for $\ell=0$ admits an analytical solution. The linearly independent solutions are $d R_{0} / d r=0$ and $d R_{0} / d r=c /\left(r^{2} f\right)$, and the constant $c$ is determined by the junction conditions. The source of our troubles is the factor of $f$ in the denominator. Because $f=0$ at either horizon, a solution that is regular at $r=r_{e}$ is necessarily singular at $r=r_{c}$, and vice versa. In pure de Sitter spacetime, the field can be made regular at $r=r_{c}$ by adopting the zero solution for $r>r_{0}$, but the internal solution will then diverge as $r^{-2}$ near $r=0$. These problems go away when there is no cosmological horizon; with anti de Sitter asymptotics the external solution can be set equal to $c /\left(r^{2} f\right)$, which decays as $r^{-4}$ when $r \rightarrow \infty$. 
Electromagnetic self-force on a static charge in Schwarzschild-de Sitter spacetimes 13

Because the singularity in the $\ell=0$ mode of the scalar field cannot be cured by the other modes, we conclude that a static scalar charge produces a field that necessarily diverges at one of the horizons in Schwarzschild-de Sitter spacetime. As we have seen, the pathology extends to pure de Sitter spacetime, but it is avoided in Schwarzschild-anti de Sitter and pure anti-de Sitter spacetimes. Changing the scalarfield equation by introducing a nonminimal coupling to the curvature also offers a way out.

\section{Acknowledgments}

This work was supported by the Natural Sciences and Engineering Research Council of Canada.

\section{References}

[1] A. G. Smith and C. M. Will, Force on a static charge outside a Schwarzschild black hole, Phys. Rev. D 22, 1276 (1980).

[2] L. M. Burko, Y. T. Liu, and Y. Soen, Self-force on charges in the spacetime of spherical shells, Phys. Rev. D 63, 024015 (2000), arXiv:gr-qc/0008065.

[3] B. S. DeWitt and R. W. Brehme, Radiation damping in a gravitational field, Ann. Phys. (N.Y.) 9, 220 (1960).

[4] E. Poisson, A. Pound, and I. Vega, The motion of point particles in curved spacetime, Living Rev. Rel. 14, 7 (2011), arXiv:1102.0529.

[5] L. Barack and N. Sago, Gravitational self-force on a particle in eccentric orbit around a Schwarzschild black hole, Phys. Rev. D 81, 084021 (2010), arXiv:1002.2386.

[6] P. Diener, I. Vega, B. Wardell, and S. Detweiler, Self-consistent orbital evolution of a particle around a Schwarzschild black hole, Phys. Rev. Lett. 108, 191102 (2012), arXiv:1112.4821.

[7] N. Warburton, S. Akcay, L. Barack, J. R. Gair, and N. Sago, Evolution of inspiral orbits around a Schwarzschild black hole, Phys. Rev. D 85, 061501 (2012), arXiv:1111.6908.

[8] W. Unruh, Self force on charged particles, Proc. Roy. Soc. London A348, 447 (1976).

[9] K. Shankar and B. F. Whiting, Self force of a static electric charge near a Schwarzschild Star, Phys. Rev. D76, 124027 (2007), arXiv:0707.0042.

[10] S. Isoyama and E. Poisson, Self-force as probe of internal structure, Class.Quant.Grav. 29, 155012 (2012), arXiv:1205.1236.

[11] T. D. Drivas and S. E. Gralla, Dependence of Self-force on Central Object, Class. Quant. Grav. 28, 145025 (2011), arXiv:1009.0504.

[12] M. Casals, E. Poisson, and I. Vega, Regularization of static self-forces, Phys. Rev. D 86, 064033 (2012), arXiv:1206.3772

[13] L. Barack, Y. Mino, H. Nakano, A. Ori, and M. Sasaki, Calculating the gravitational self-force in Schwarzschild spacetime, Phys. Rev. Lett. 88, 091101 (2002), arXiv:gr-qc/0111001.

[14] L. Barack and A. Ori, Regularization parameters for the self-force in Schwarzschild spacetime: Scalar case, Phys. Rev. D 66, 084022 (2002), arXiv:gr-qc/0204093.

[15] L. Barack and A. Ori, Regularization parameters for the self-force in Schwarzschild spacetime. II. Gravitational and electromagnetic cases, Phys. Rev. D 67, 024029 (2003), arXiv:grqc/0209072.

[16] L. M. Diaz-Rivera, E. Messaritaki, B. F. Whiting, and S. Detweiler, Scalar field self-force effects on orbits about a Schwarzschild black hole, Phys. Rev. D 70, 124018 (2004), arXiv:grqc/0410011. 